\title{
Atomic resolution structure determination by cryo-EM - where are the limits?
}

\author{
Holger Stark \\ Max-Planck-Institute for biophysical chemistry, Goettingen, Germany; \\ hstark1@gwdg.de
}

Single particle cryo electron microscopy (cryo-EM) has developed into a powerful technique to determine 3D structures of large macromolecular complexes. Due to improvements in instrumentation and computational image analysis, the number of highresolution structures is steadily increasing. The method cannot only be used to determine high-resolution structures but also to study the dynamic behavior of macromolecular complexes and thus represents a very complementary method to X-ray crystallography. Furthermore, the maximum attainable resolution by cryo-EM has constantly improved in recent years. Most of the high-resolution structures are still in the 3 Angstrom resolution regime but some have even crossed the 2 Angstrom barrier. We have recently installed a new prototype electron microscope which is equipped with a monochromator and a next-generation spherical aberration corrector. This microscope is optically superior to the currently commercially available instruments and can therefore be used to test the resolution limits in cryo-EM. We have used the test specimen apoferritin to determine its structure at 1.25 Angstrom resolution which is sufficient to visualize for the first time individual atoms clearly separated in the density map without the need for computational beam tilt corrections.

Recently, we managed to use this microscope not only to improve the resolution of the very stable and rigid protein apoferritin. We also obtained significant improvement in resolution for other more dynamic macromolecular complexes for which one could have expected that the microscope itself may not be a major resolution limiting factor.

In current high-resolution cryo-EM structures less water molecules become visible compared to X-ray crystallographic structures at nominally the same level of resolution. The number of water molecules that can be reliably build into the EM density is also not independent from the image processing software used for the three-dimensional reconstruction. We made a first attempt to use the number of water molecules that can be build into a 3D structure as a quality criterium for cryo-EM data since until now such highresolution quality estimators are entirely missing in the cryo-EM field.

We are currently upgrading our microscope with an energy filter and a faster direct pixel detector. This will not only improve throughput but also the maximum attainable resolution even further. Therefore I will address the question of how much the resolution in cryo-EM can still be realistically improved and how this compares to X-ray crystallography.

Keywords: cryo-EM, atomic resolution, aberration correction, monochromator 